\begin{tabular}{|c|l|}
\hline Title & Theory of photoexcited carrier relaxation across the energy gap of phase ordered materials \\
\hline Author(s) & Ono, Shota; Shima, Hiroyuki; Toda, Y asunori \\
\hline Citation & $\begin{array}{l}\text { Physical Review B, 86/10), 104512 } \\
\text { https://doi.org/10.1103/PhysRevB.86.104512 }\end{array}$ \\
\hline Issue Date & 2012-09-01 \\
\hline Doc URL & http://hdl.handle.net/2115/50422 \\
\hline Rights & ○2012A A merican Physical Society \\
\hline Type & article \\
\hline File Information & PRB86-10_104512.pdf \\
\hline
\end{tabular}

Instructions for use 


\title{
Theory of photoexcited carrier relaxation across the energy gap of phase-ordered materials
}

\author{
Shota Ono, ${ }^{1,2, *}$ Hiroyuki Shima, ${ }^{2,3}$ and Yasunori Toda ${ }^{2}$ \\ ${ }^{1}$ Department of Physics, Graduate School of Engineering, Yokohama National University, Yokohama 240-8501, Japan \\ ${ }^{2}$ Division of Applied Physics, Faculty of Engineering, Hokkaido University, Sapporo, Hokkaido 060-8628, Japan \\ ${ }^{3}$ Department of Environmental Sciences and Interdisciplinary Graduate School of Medicine and Engineering, \\ University of Yamanashi, 4-4-37, Takeda, Kofu, Yamanashi 400-8510, Japan
}

(Received 12 February 2012; revised manuscript received 29 August 2012; published 26 September 2012)

\begin{abstract}
We develop a theory to describe the energy relaxation of photoexcited carriers in low-temperature ordered states with a band-gap opening. Carrier relaxation time $\tau$ near and below transition temperature $T_{\mathrm{c}}$ is formulated by examining the contributions from different carrier-phonon scatterings to the relaxation rate. Transverse acoustic phonon modes are found to play a crucial role in carrier relaxation; their heat capacity determines the $\tau$ divergence near $T_{\mathrm{c}}$. Remarkable agreements with the theory and experimental data on two different materials which exhibit contrasting $\tau(T)$ behaviors are also demonstrated.
\end{abstract}

DOI: 10.1103/PhysRevB.86.104512

PACS number(s): 74.25.Gz, 78.47.-p, 63.20.kk, 71.45.Lr

\section{INTRODUCTION}

The nonequilibrium dynamics of photoexcited carriers in solids has attracted considerable research interest in the field of condensed matter physics. These dynamics are governed by multiple scatterings of carriers and the energy transfer to the phonon field, both of which are quantified by the carrier relaxation time $\tau_{\exp }$ and its temperature $(T)$ dependence. Usually the anomalous $T$ dependence of $\tau_{\exp }$ is observed in many gapped systems, for example, phaseordered systems showing an energy gap opening in the electron band below the transition temperature $T_{\mathrm{c}}$. It has been found that a wide variety of superconductors ${ }^{1-13}$ and density-wave compounds ${ }^{14-19}$ show diverging behavior of $\tau_{\text {exp }}$ near $T_{\mathrm{c}}$, as confirmed by femtosecond time-resolved optical spectroscopy. ${ }^{1,4,5,7-17,19}$ The divergence of $\tau_{\exp }$ in these gapped systems is believed to result from a recursive energy transfer between electrons and phonons. Photoexcited electrons having high energy emit a number of phonons through relaxation from above to below the energy gap. Conversely, relaxed electrons can be reexcited above the gap by absorbing phonon energy. This phonon emission-reabsorption process becomes efficient near $T_{\mathrm{c}}$ because of the small gap energy, and thus it suppresses the relaxation of carriers, extending $\tau_{\exp }$ significantly. This anomalous phonon-mediated relaxation in gapped systems is called the phonon bottleneck effect. This bottleneck enables the reproduction of these experimental observations of $\tau_{\exp }$ in various gapped systems that exhibit $\tau_{\text {exp }}$ divergence at $T_{\mathrm{c}}$.

There exists, however, a distinct class of gapped systems such as $\mathrm{Tl}$-based superconductors ${ }^{2,6}$ and $\mathrm{C}_{60}$-related materials ${ }^{3,18}$ that, instead of showing $\tau_{\exp }$ divergence at $T_{\mathrm{c}}$, show monotonic increases in $\tau_{\exp }$ with cooling across $T_{\mathrm{c}}$. In light of the bottleneck, the monotonic variation in $\tau_{\exp }$ near $T_{\mathrm{c}}$ appears controversial, leading to the questions of why the $\tau_{\exp }$ divergence vanishes in a portion of gapped systems despite the well-defined energy gap formation at the Fermi level and whether the bottleneck concept is completely invalid in these gapped systems. Theoretical studies have been unable to satisfactorily answer these two questions over the last decade.

In this paper, we develop a theory of photoexcited carrier relaxation dynamics with the objective of resolving the abovementioned issues. We postulate that the lack of $\tau_{\exp }$ divergence even in gapped systems can be ascribed to the presence of transverse acoustic (TA) phonon modes. Further, we state that an ensemble of TA modes in gapped systems serves as a highcapacity thermal sink, and the energy release to the sink from other phonon modes facilitates the efficient cooling of carriers, in other words, a significant reduction in $\tau_{\text {exp }}$ close to $T_{\mathrm{c}}$. The proposed theory is validated by the quantitative agreement with the experimental data of $\tau_{\text {exp }}$ 's for peanut-shaped $\mathrm{C}_{60}$ polymers and high- $T_{\mathrm{c}}$ superconductor $\mathrm{Bi}_{2} \mathrm{Sr}_{2} \mathrm{CaCu}_{2} \mathrm{O}_{8+\delta}$ (Bi2212); the first of these is a typical charge density wave (CDW) material devoid of $\tau_{\text {exp }}$ divergence.

Section II starts with a description of the phonon dynamics based on the phonon bottleneck concept (Sec. II A), followed by the derivation of the carrier relaxation time (Sec. II B). In Sec. II B1, we show the kinetic Boltzmann equation taking into account the phonon-phonon collision for gapped systems. In Secs. II B2 and II B3, we demonstrate how to calculate the collision terms and derive the expression of the anharmonic decay time $(\tau)$ below $T_{\mathrm{c}}$ (Ref. 20), respectively. Section III is devoted to the numerical results. The temperature dependence of $\tau$ is shown in Sec. III A. The comparison between our model and the experimental data is made in Sec. III B. Conclusions are presented in Sec. IV. For the explicit expression of $\tau$, in the Appendix, all collision terms are listed.

\section{THEORY}

\section{A. Description of the phonon dynamics}

First, we briefly review the conventional bottleneck concept for $\tau_{\exp }$ divergence. It is based on the assumption that photoexcited carrier relaxation in gapped systems is regulated by the anharmonic slow decay of phonons. ${ }^{1,23}$ By the absorption of a pump laser photon, electrons in the valence band are excited far above the initial states, and they rapidly accumulate in the upper end of the gap through carrier-carrier and carrier-phonon interactions. Then, the carriers emit high-energy phonons (HEPs) whose energies are higher than the gap width $2 \Delta$ to relax into the lower end of the gap. The HEPs produced can be reabsorbed to create new carriers above the gap [see Fig. 1(a)] or they can decay in an anharmonic manner into low-energy phonons (LEPs) that no longer excite carriers 
(a)
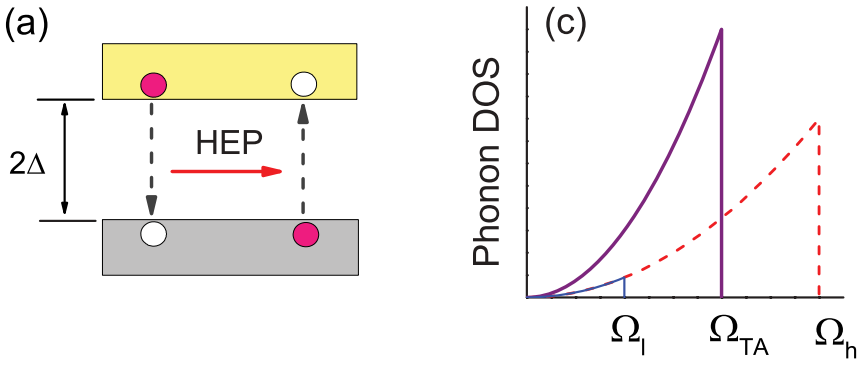

(b)
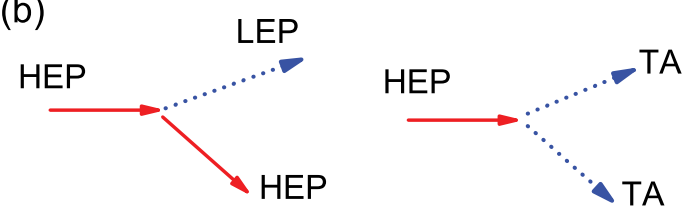

FIG. 1. (Color online) (a) Emission-reabsorption process of HEPs across the energy gap with width $2 \Delta$. (b) Diagram of threephonon scattering processes through which HEPs dissipate. Only the scatterings that involve TA modes are relevant to the lack of divergence in $\tau$. (c) Phonon density of states of TA modes with cutoff frequency $\Omega_{\mathrm{TA}}$, and those of LA modes with $\Omega_{h}$. The maximum frequency of the LEP is $\Omega_{l}$ (see text for its definition).

because their energy is lower than $2 \Delta$ [see the left-hand side panel in Fig. 1(b)]. If the reabsorption probability per unit time is much larger than the inverse of the anharmonic decay time $\left(\tau^{-1}\right)$, photoexcited carriers and HEPs settle in nearly steady states that obey the Fermi and Bose distribution functions, respectively, with temperature $T^{\prime}$ that is higher than the lattice temperature $T$ (Ref. 1). Meanwhile, LEPs obey the Bose distribution function with $T$ because they remain unperturbed after the laser pulse incident. Consequently, the carrier relaxation is dominated by the energy transfer from HEPs to LEPs, that is, $\tau_{\exp } \simeq \tau$, which is described by the time evolution of the two temperatures, that is, $T^{\prime}=T^{\prime}(t)$ and $T=T(t)$. In this way, with the help of the bottleneck concept, the carrier relaxation across the gap can be mapped into the phonon relaxation dynamics.

It should be emphasized that in the conventional bottleneck concept, only longitudinal acoustic (LA) phonon modes are considered. Here, we point out the unnoticed but important role of TA phonon modes in the HEP's decay [see the right-hand side panel in Fig. 1(b)]. Because TA phonon modes generate no density modulation in the lattice, they cannot interact with photoexcited carriers within the deformation potential theory. This fact implies that TA modes serve as a thermal receiver into which HEPs can dissipate, ${ }^{24}$ as a result of which the $\tau$ divergence vanishes even at $T_{\mathrm{c}}$. Below, we prove that this holds true in certain gapped systems.

Before going into the derivation of $\tau$, let us mention how our theory is compared to the experiment. In a pump probe experiment, a transient reflectivity change should be measured by probe pulses. By absorbing an intense pump pulse, carriers are excited far above the initial state: This leads to a change in the reflectivity of the probe pulse with a delay to the pump pulse. The magnitude of the change is proportional to the number of the nonequilibrium carriers. ${ }^{1}$ Due to the phonon bottleneck effect, the nonequilibrium carriers relax down to states near the gap via electron-electron and electron- phonon scatterings, resulting in quasi-equilibrium populations of carriers and HEPs. They then reach equilibrium by HEP decay. After the quasi-equilibrium, the rate of decrease in the number of carriers is equivalent to the decay rate of HEPs: The latter is regulated by the time evolution of $T^{\prime}$ and $T$. Thus, the reflectivity change is time dependent, which can be described by a two-temperature model.

\section{B. Derivation of the relaxation time based on the phonon bottleneck concept \\ 1. Phonon-phonon collisions}

To formulate the anharmonic decay time $\tau$ of HEPs, we consider the time evolution of the phonon distribution function. Following the above discussion, we divide phonon excitations into two groups. One group consists of HEPs $\left(\Omega_{l}<\omega_{q \text {,LA }}<\right.$ $\Omega_{h}$ ) in equilibrium temperature at $T^{\prime}$ that can participate in the reabsorption process. The other group involves TA modes $\left(\omega_{q, \mathrm{TA}}<\Omega_{\mathrm{TA}}\right)$ and LEPs $\left[\omega_{q, \mathrm{LA}}<2 \Delta / \hbar\left(\equiv \Omega_{l}\right)\right]$ in equilibrium at $T$ that do not participate in the reabsorption process. Here, $\omega_{q, \mathrm{TA}}$ and $\omega_{q, \mathrm{LA}}$ represent the phonon dispersion relations, where $\boldsymbol{q}$ is the wave vector. $\Omega_{h}, \Omega_{l}$, and $\Omega_{\mathrm{TA}}$ are the cutoff frequencies for the HEP, LEP, and TA modes within the Debye approximation, respectively. The distribution functions for the two groups are given by $n\left(\omega_{\boldsymbol{q}, j}\right)=\left\{\exp \left[\left(\hbar \omega_{\boldsymbol{q}, j}\right) /\left(k_{B} x\right)\right]-1\right\}^{-1}$, with an appropriate variable $x=T$ or $T^{\prime}$.

The rate of change in $n\left(\omega_{\boldsymbol{q}, j}\right)$ due to phonon-phonon collisions is written as ${ }^{26}$

$$
\frac{\partial n\left(\omega_{\boldsymbol{q}, j}\right)}{\partial t}=J_{\mathrm{col}}\left[n\left(\omega_{\boldsymbol{q}, j}\right)\right] .
$$

The collision integral $J_{\text {col }}\left[n\left(\omega_{\boldsymbol{q}, j}\right)\right]$ describes three-phonon scattering, and it is defined by

$$
J_{\mathrm{col}}\left[n\left(\omega_{0}\right)\right]=\frac{2 \pi}{\hbar N^{2}} \sum_{\boldsymbol{q}_{1}, \boldsymbol{q}_{2}, j_{1}, j_{2}}\left|w_{i \rightarrow f}\right|^{2}\left(\frac{1}{2} S_{A}+S_{B}\right),
$$

where $S_{A}=\left\{\left[n\left(\omega_{0}\right)+1\right] n\left(\omega_{1}\right) n\left(\omega_{2}\right)-n\left(\omega_{0}\right)\left[n\left(\omega_{1}\right)+1\right] \times\right.$ $\left.\left[n\left(\omega_{2}\right)+1\right]\right\} \delta\left(\hbar \omega_{0}-\hbar \omega_{1}-\hbar \omega_{2}\right) \quad$ and $\quad S_{B}=\left\{\left[n\left(\omega_{0}\right)+1\right]\right.$ $\left.\left[n\left(\omega_{1}\right)+1\right] n\left(\omega_{2}\right)-n\left(\omega_{0}\right) n\left(\omega_{1}\right)\left[n\left(\omega_{2}\right)+1\right]\right\} \delta\left(\hbar \omega_{2}-\hbar \omega_{0}-\hbar \omega_{1}\right)$. Here $\omega_{s}(s=0,1,2)$ is an abbreviation of $\omega_{\boldsymbol{q}_{s}, j_{s}}$, and $w_{i \rightarrow f}$ is the matrix element between the initial and the final state. ${ }^{27}$

Note that $J_{\text {col }}$ regulates the time evolution of the total energy through the relationship

$$
\begin{gathered}
\frac{\partial}{\partial t}\left(E_{\mathrm{LA}}+E_{\mathrm{TA}}\right)=K_{\mathrm{LA}}+K_{\mathrm{TA}}, \\
K_{j}=\sum_{q(j)} \hbar \omega_{\boldsymbol{q}, j} J_{\mathrm{col}}, \quad(j=\mathrm{LA}, \mathrm{TA}),
\end{gathered}
$$

where the summation of Eq. (4) runs over $\boldsymbol{q}$ 's satisfying $0<$ $\omega_{q, \mathrm{LA}}<\Omega_{l}$ for $j=\mathrm{LA}$ and $0<\omega_{q, \mathrm{TA}}<\Omega_{\mathrm{TA}}$ for $j=\mathrm{TA}$. The energy $E_{j}$ is defined by

$$
E_{j}=\sum_{\boldsymbol{q}(j)} \hbar \omega_{\boldsymbol{q}, j} n\left(\omega_{\boldsymbol{q}, j}\right)=\int_{0}^{y} \hbar \omega n(\omega) \rho_{j}(\omega) d \omega,
$$


where $y=\Omega_{\mathrm{TA}}\left(\Omega_{l}\right)$ for $j=\mathrm{TA}$ (LA). $\rho_{j}$ is the density of states defined by

$$
\rho_{j}(\omega)=\alpha_{j} \omega^{2} \theta(z-\omega), \quad\left(\alpha_{j}=\frac{3 v_{j} N}{z^{3} \sum_{j^{\prime}} v_{j^{\prime}}}\right),
$$

where $z=\Omega_{\mathrm{TA}}\left(\Omega_{h}\right)$ for $j=\mathrm{TA}(\mathrm{LA}), v_{j^{\prime}}$ is the number of phonon branches participating in the three-phonon scattering, $N$ is the number of unit cells, and $\theta$ is the Heaviside step function; the total density of states is given by $\rho=\rho_{\mathrm{TA}}+\rho_{\mathrm{LA}}$ [see Fig. 1(c)], satisfying the normalization condition $1=$ $\int \rho(\omega) d \omega / N$. The Debye approximation we have used in Eq. (6) is valid when $k_{B} T<\hbar \Omega_{\mathrm{TA}}\left(<\hbar \Omega_{h}\right)$, which holds for many gapped systems below $T_{\mathrm{c}}$. We simplify $n(\omega)$ in Eq. (5) as $k_{B} T / \hbar \omega^{1}$ to obtain $E_{j}=C_{j} T$, where $C_{j}=k_{B} \alpha_{j} y^{3} / 3\left(k_{B}\right.$ is the Boltzmann constant).

Substituting Eq. (2) into the right-hand side of Eq. (4) and replacing the summations by integrals, we obtain

$$
\begin{aligned}
K_{\mathrm{LA}}= & \frac{2 \pi w_{1}^{2}}{\hbar^{2} N^{2}} \int_{0}^{\Omega_{l}} d \omega \int d \omega^{\prime}\left[S_{a}\left(T, T^{\prime}\right) \rho_{\mathrm{LA}}(\omega) \rho_{\mathrm{LA}}\left(\omega^{\prime}\right)\right. \\
& \times \rho_{\mathrm{LA}}\left(\omega-\omega^{\prime}\right)+S_{b}\left(T, T^{\prime}\right) \rho_{\mathrm{LA}}(\omega) \rho_{\mathrm{LA}}\left(\omega^{\prime}\right) \\
& \left.\times \rho_{\mathrm{LA}}\left(\omega+\omega^{\prime}\right)\right], \\
K_{\mathrm{TA}}= & \frac{2 \pi w_{2}^{2}}{\hbar^{2} N^{2}} \int_{0}^{\Omega_{\mathrm{TA}}} d \omega \int d \omega^{\prime}\left[S_{a}\left(T, T^{\prime}\right) \rho_{\mathrm{TA}}(\omega) \rho\left(\omega^{\prime}\right)\right. \\
& \left.\times \rho\left(\omega-\omega^{\prime}\right)+S_{b}\left(T, T^{\prime}\right) \rho_{\mathrm{TA}}(\omega) \rho\left(\omega^{\prime}\right) \rho\left(\omega+\omega^{\prime}\right)\right],
\end{aligned}
$$

where $\rho(\omega)=\rho_{\mathrm{LA}}(\omega)+\rho_{\mathrm{TA}}(\omega)$ and

$$
\begin{aligned}
S_{a}\left(T, T^{\prime}\right)= & \frac{\hbar \omega}{2}\left\{[n(\omega)+1] n\left(\omega^{\prime}\right) n\left(\omega-\omega^{\prime}\right)-n(\omega)\left[n\left(\omega^{\prime}\right)+1\right]\right. \\
& \left.\times\left[n\left(\omega-\omega^{\prime}\right)+1\right]\right\},
\end{aligned}
$$

$S_{b}\left(T, T^{\prime}\right)=\hbar \omega\left\{[n(\omega)+1]\left[n\left(\omega^{\prime}\right)+1\right] n\left(\omega+\omega^{\prime}\right)-n(\omega) n\left(\omega^{\prime}\right)\right.$

$$
\left.\times\left[n\left(\omega+\omega^{\prime}\right)+1\right]\right\} .
$$

Here the first term in the brackets in Eqs. (7) and (8) are the contributions from the three-phonon processes (i.e., decays of two phonons with frequencies $\omega^{\prime}$ and $\omega-\omega^{\prime}$ followed by the creation of a phonon mode with $\omega$ and vice versa). The second term is due to the decay of a phonon with $\omega+\omega^{\prime}$ followed by the creation of two phonon modes with $\omega$ and $\omega^{\prime}$ and vice versa. Equation (8) describes the three-phonon processes in which both the LA and TA modes are involved, whereas Eq. (7) describes the processes in which only the LA modes are involved. To derive Eqs. (7) and (8), we assumed that the matrix element is momentum independent, that is, $w_{i \rightarrow f} \equiv w_{1}$ (or $\left.w_{2}\right)=$ const. when TA modes are absent from (join in) the three-phonon scattering.

\section{Calculation of the collision integral}

We now derive the expression of $K_{\mathrm{TA}}$ that appears in Eq. (8). For this purpose, the integration in Eq. (8) should be performed. The integrand in Eq. (8) has the products of the Bose distribution functions (BDFs): $n(\omega), n\left(\omega^{\prime}\right)$, and $n(\omega \pm$ $\left.\omega^{\prime}\right)$. The temperature endowed in each BDF depends upon the phonon mode, as given by $n(\omega)=\left\{\exp \left[(\hbar \omega) /\left(k_{B} x\right)\right]-1\right\}^{-1}$ $\left(x=T\right.$ or $\left.T^{\prime}\right)$. The phonon mode with frequency $\omega^{\prime}\left(\omega \pm \omega^{\prime}\right)$
TABLE I. Phonon mode combinations for three-phonon processes in gapped systems.

\begin{tabular}{cccccccc}
\hline \hline & $\omega$ & $\omega^{\prime}$ & $\omega-\omega^{\prime}$ & & $\omega$ & $\omega^{\prime}$ & $\omega+\omega^{\prime}$ \\
\hline$K_{A}^{(1)}$ & TA & LA & LA & $K_{B}^{(1)}$ & TA & LA & LA \\
$K_{A}^{(2)}$ & TA & TA & LA & $K_{B}^{(2)}$ & TA & TA & LA \\
$K_{A}^{(3)}$ & TA & LA & TA & $K_{B}^{(3)}$ & TA & LA & TA \\
\hline \hline
\end{tabular}

is either the TA or the LA mode; there are six patterns, as listed in Table I. In Table I, there is no case in which both phonons with $\omega^{\prime}$ and $\omega \pm \omega^{\prime}$ are in the TA mode; this is because the integrand in Eq. (8) is always zero if all the distribution functions - $n(\omega), n\left(\omega^{\prime}\right)$, and $n\left(\omega \pm \omega^{\prime}\right)$-are associated with the same temperature $T$. Thus, the collision integral (8) can be decomposed as

$$
K_{\mathrm{TA}}=\sum_{j=1,2,3}\left[K_{A}^{(j)}+K_{B}^{(j)}\right]
$$

where $\sum_{i=1}^{3} K_{A}^{(j)}\left(\sum_{i=1}^{3} K_{B}^{(j)}\right)$ is the integral of the first (second) term of the bracket in Eq. (8). Each $K_{A(B)}^{(j)}$ is the contribution from the three-phonon processes shown in Table I.

The following deals with a derivation of $K_{A}^{(1)}$, as an example. Repeating the similar derivation, we can obtain expressions for $K_{A}^{(j)}(j=2,3), K_{B}^{(j)}(j=1,2,3)$, and $K_{\mathrm{LA}}$ by taking care of the domains in the double integral in Eqs. (7) and (8) (see the Appendix for the expressions).

To derive $K_{A}^{(1)}$, let us consider the following three cases: (i) $2 \Omega_{l}<\Omega_{\mathrm{TA}}$, (ii) $\Omega_{l}<\Omega_{\mathrm{TA}}<2 \Omega_{l}$, and (iii) $\Omega_{\mathrm{TA}}<\Omega_{l}$. In case (i), because the LA phonon modes with $\omega^{\prime}$ and/or $\omega-\omega^{\prime}$ obey the BDF characterized by temperature $T^{\prime}$ that is higher than the lattice temperature $T$, the expression of the integrand in Eq. (8) depends on the magnitude of the frequencies $\omega$ and $\omega^{\prime}$. Depending on $\omega$ and $\omega^{\prime}$, the domain on the $\omega$ - $\omega^{\prime}$ plane in Eq. (8) can be decomposed into three pieces, $M_{1}, M_{2}$, and $M_{3}$ [see Fig. 2(a)], so that $K_{A}^{(1)}=\sum_{j=1,2,3} Q_{A}^{(1)}(j)$, where $Q_{A}^{(1)}(j)$ is equal to the integral over the domain $M_{j}$. In the domain represented by $M_{1}$, the phonon modes with $\omega^{\prime}$ and $\omega-\omega^{\prime}$ obey the BDF with $T^{\prime}$ and $T$, respectively (i.e., $n\left(\omega^{\prime}\right) \simeq k_{B} T^{\prime} /\left(\hbar \omega^{\prime}\right)$ (a) $2 \Omega_{l}<\Omega_{\mathrm{TA}}$

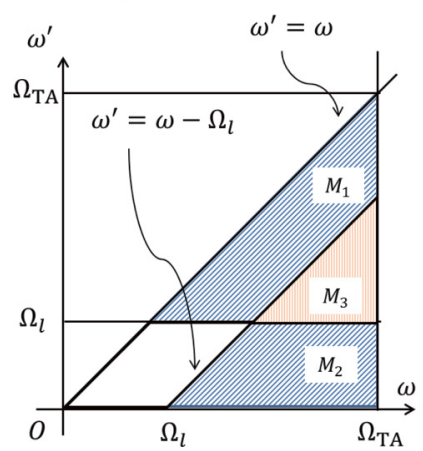

(b) $\Omega_{l}<\Omega_{h}<2 \Omega_{l}$

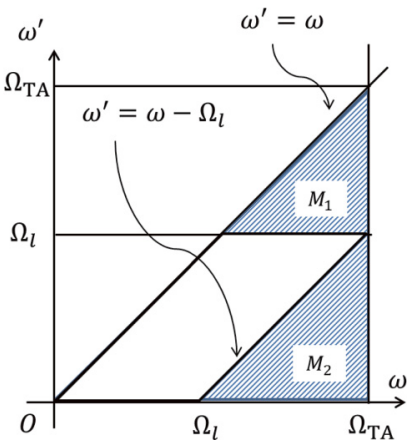

FIG. 2. (Color online) Domains on the $\omega-\omega^{\prime}$ plane appearing in the double integral $K_{\mathrm{TA}}^{(1)}$ given by Eq. (8) for (a) $2 \Omega_{l}<\Omega_{\mathrm{TA}}$ and (b) $\Omega_{l}<\Omega_{\mathrm{TA}}<2 \Omega_{l}$. 
and $\left.n\left(\omega-\omega^{\prime}\right) \simeq k_{B} T /\left[\hbar\left(\omega-\omega^{\prime}\right)\right]\right)$. Then, $Q_{A}^{(1)}$ is written by

$$
\begin{aligned}
Q_{A}^{(1)}(1)= & \frac{2 \pi w_{2}^{2}}{\hbar^{2} N^{2}}\left(\int_{\Omega_{l}}^{2 \Omega_{l}} d \omega \int_{\Omega_{l}}^{\omega} d \omega^{\prime}+\int_{2 \Omega_{l}}^{\Omega_{\mathrm{TA}}} d \omega \int_{\omega-\Omega_{l}}^{\omega} d \omega^{\prime}\right) \\
& \times \mu\left(\omega, \omega^{\prime}\right) S_{a}\left(T, T^{\prime}\right) \\
= & w_{2}^{2} \gamma_{1} G_{A}^{(1)}(1) T\left(T^{\prime}-T\right), \\
G_{A}^{(1)}(1)= & \frac{71}{168} \Omega_{l}^{7}+\frac{1}{120}\left(6 \Omega_{l}^{2} \Omega_{\mathrm{TA}}^{5}-10 \Omega_{l}^{3} \Omega_{\mathrm{TA}}^{4}\right. \\
& \left.+5 \Omega_{l}^{4} \Omega_{\mathrm{TA}}^{3}-72 \Omega_{l}^{7}\right),
\end{aligned}
$$

where $\mu\left(\omega, \omega^{\prime}\right)=\rho_{\mathrm{TA}}(\omega) \rho_{\mathrm{LA}}\left(\omega^{\prime}\right) \rho_{\mathrm{LA}}\left(\omega-\omega^{\prime}\right)$. In domain $M_{2}$, the phonon mode with $\omega^{\prime}\left(\omega-\omega^{\prime}\right)$ obeys the BDF with $T\left(T^{\prime}\right)$. Then, $Q_{A}^{(2)}$ is written by

$$
\begin{aligned}
& Q_{A}^{(1)}(2)= \frac{2 \pi w_{2}^{2}}{\hbar^{2} N^{2}}\left(\int_{\Omega_{l}}^{2 \Omega_{l}} d \omega \int_{0}^{\omega-\Omega_{l}} d \omega^{\prime}+\int_{2 \Omega_{l}}^{\Omega_{\mathrm{TA}}} d \omega \int_{0}^{\Omega_{l}} d \omega^{\prime}\right) \\
& \times \mu\left(\omega, \omega^{\prime}\right) S_{a}\left(T, T^{\prime}\right) \\
&= w_{2}^{2} \gamma_{1} G_{A}^{(1)}(2) T\left(T^{\prime}-T\right), \\
& G_{A}^{(1)}(2)=G_{A}^{(1)}(1) .
\end{aligned}
$$

In domain $M_{3}$, both the phonon modes with $\omega^{\prime}$ and $\omega-\omega^{\prime}$ obey the BDF with $T^{\prime}$. Then

$$
\begin{aligned}
Q_{A}^{(1)}(3) & =\frac{2 \pi w_{2}^{2}}{\hbar^{2} N^{2}}\left(\int_{2 \Omega_{l}}^{\Omega_{\mathrm{TA}}} d \omega \int_{\Omega_{l}}^{\omega-\Omega_{l}} d \omega^{\prime}\right) \mu\left(\omega, \omega^{\prime}\right) S_{a}\left(T, T^{\prime}\right) \\
& =w_{2}^{2} \gamma_{1} G_{A}^{(1)}(3) T^{\prime}\left(T^{\prime}-T\right), \\
G_{A}^{(1)}(3) & =\frac{1}{420}\left(5 \Omega_{\mathrm{TA}}^{7}-42 \Omega_{l}^{2} \Omega_{\mathrm{TA}}^{5}+35 \Omega_{l}^{3} \Omega_{\mathrm{TA}}^{4}+144 \Omega_{l}^{7}\right) .
\end{aligned}
$$

In case (ii), the domain in Eq. (8) can be decomposed into two pieces, $M_{1}$ and $M_{2}$, as shown in Fig. 2(b). Therefore, we obtain $K_{A}^{(1)}=\sum_{j=1,2} Q_{A}^{(1)}(j)$, where the definition of $Q_{A}^{(1)}(j)$ is the same as that in case (i). In domain $M_{1}$, the phonon mode with $\omega^{\prime}$ obeys the BDF with $T^{\prime}$, whereas that with $\omega-\omega^{\prime}$ obeys the BDF with $T$. Then, $Q_{A}^{(1)}(1)$ is written as

$$
\begin{aligned}
Q_{A}^{(1)}(1) & =\frac{2 \pi w_{2}^{2}}{\hbar^{2} N^{2}}\left(\int_{\Omega_{l}}^{\Omega_{\mathrm{TA}}} d \omega \int_{\Omega_{l}}^{\omega} d \omega^{\prime}\right) \mu\left(\omega, \omega^{\prime}\right) S_{a}\left(T, T^{\prime}\right) \\
& =w_{2}^{2} \gamma_{1} G_{A}^{(1)}(1) T\left(T^{\prime}-T\right), \\
G_{A}^{(1)}(1) & =\frac{1}{168}\left(\Omega_{\mathrm{TA}}^{7}-7 \Omega_{l}^{2} \Omega_{\mathrm{TA}}^{4}+7 \Omega_{l}^{4} \Omega_{\mathrm{TA}}^{3}-\Omega_{l}^{7}\right)
\end{aligned}
$$

In domain $M_{2}$, the phonon modes with $\omega^{\prime}$ and $\omega-\omega^{\prime}$ obey the BDF with $T$ and $T^{\prime}$, respectively. Then

$$
\begin{gathered}
Q_{A}^{(1)}(2)=\frac{2 \pi w_{2}^{2}}{\hbar^{2} N^{2}}\left(\int_{\Omega_{l}}^{\Omega_{\mathrm{TA}}} d \omega \int_{0}^{\omega-\Omega_{l}} d \omega^{\prime}\right) \mu\left(\omega, \omega^{\prime}\right) S_{a}\left(T, T^{\prime}\right) \\
=w_{2}^{2} \gamma_{1} G_{A}^{(1)}(2) T\left(T^{\prime}-T\right), \\
G_{A}^{(1)}(2)=G_{A}^{(1)}(1) .
\end{gathered}
$$

In case (iii), because both phonon modes with $\omega^{\prime}$ and $\omega-\omega^{\prime}$ obey the BDF with $T$, there is no three-phonon process such that $K_{A}^{(1)}$ has a finite value, [i.e., $K_{A}^{(1)}=0$ ].
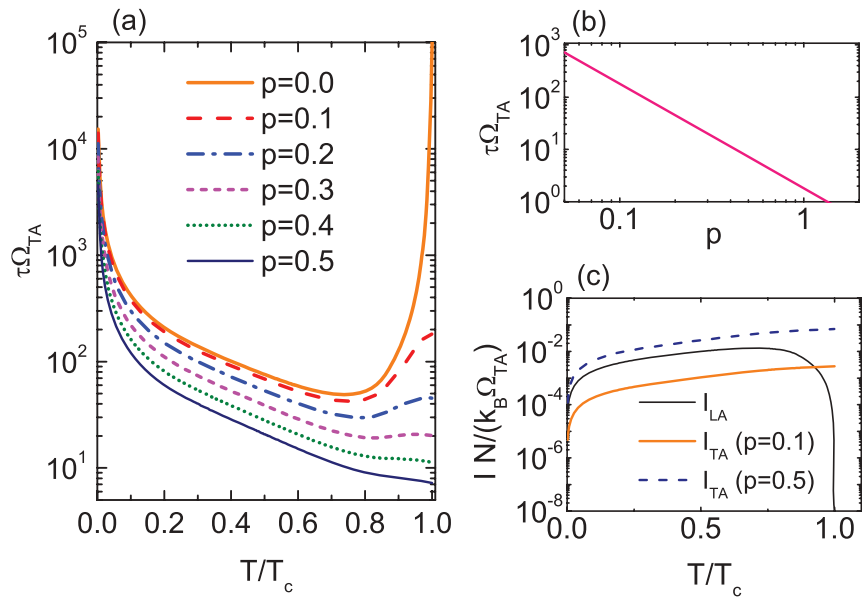

FIG. 3. (Color online) (a) Numerical result of $\tau(T)$ based on Eq. (22). See text for detailed numerical conditions. (b) Inverse square law of $\tau$ at $T=T_{\mathrm{c}}$ with respect to $p$. (c) $T$ dependence of $I_{\mathrm{LA}}$ and $I_{\mathrm{TA}}$ defined by Eqs. (24) and (25). Because $I_{\mathrm{TA}}$ differs from zero at $T=T_{\mathrm{c}}$, the $\tau$ divergence at $T_{\mathrm{c}}$ is strongly suppressed.

\section{Relaxation time}

As a result, Eq. (3) is rewritten as ${ }^{28}$

$$
\frac{\partial T(t)}{\partial t}=\frac{1}{\tau}\left[T^{\prime}-T(t)\right], \quad \tau=\frac{C_{\mathrm{LA}}+C_{\mathrm{TA}}}{I_{\mathrm{LA}}+I_{\mathrm{TA}}},
$$

using the definitions

$$
I_{j}=\frac{K_{j}}{T^{\prime}-T}, \quad(j=\mathrm{LA}, \mathrm{TA}),
$$

and

$$
\begin{gathered}
I_{\mathrm{LA}}=w_{1}^{2} \gamma_{0}\left(V_{A} T+V_{B} T^{\prime}\right), \\
I_{\mathrm{TA}}=w_{2}^{2} \gamma_{1}\left(V_{C} T+V_{D} T^{\prime}\right)+w_{2}^{2} \gamma_{2} V_{E} T,
\end{gathered}
$$

where $\gamma_{k}=2 \pi k_{B}^{2} \alpha_{\mathrm{LA}}^{3-k} \alpha_{\mathrm{TA}}^{k} /\left(\hbar^{3} N^{2}\right) . T^{\prime}$ is given by $k_{B} T^{\prime}=$ $-\Delta / \ln \left\{\epsilon+\exp \left[-\Delta /\left(k_{B} T\right)\right]\right\}$, where $\epsilon$ is the dimensionless photoexcitation energy. ${ }^{1} V_{X}(X=A$ to $E)$ in Eqs. (24) and (25) are functions of $\Omega_{l}, \Omega_{\mathrm{TA}}$, and $\Omega_{h}$. Equation (22) is the main finding of this study because it clearly shows the contribution of TA phonon modes to carrier relaxation.

\section{RESULTS AND DISCUSSIONS}

\section{A. Crossover from diverging to nondiverging behaviors}

Figure 3(a) shows the $T$ dependence of $\tau$ that we have formulated in Eq. (22). The parameter $p\left(\equiv w_{2} / w_{1}\right)$ is the coupling strength through which the HEPs decay into TA modes, and it is tuned from 0 to 0.5 in increments of 0.1 . With an increase in $p$, the magnitude of $\tau$ decreases over the entire $T$ range. When $p$ is smaller (larger) than $\sim 0.3, \tau$ increases (decreases) as $T$ approaches $T_{\mathrm{c}}$ from below. The most significant phenomenon is the drastic reduction in $\tau$ at $T_{\mathrm{c}}$ with increasing $p$. In fact, $\tau$ at $T_{\mathrm{c}}$ is inversely proportional to $p^{2}$, as shown in Fig. 3(b). These results indicate that the lack of $\tau$ divergence at $T_{\mathrm{c}}$ is attributable to efficient phonon-phonon coupling between the HEP and the TA mode characterized by $p$. 
To explain the microscopic mechanism for the lack of divergence, in Fig. 3(c), we show the $T$ dependence of the magnitude of $I_{\mathrm{LA}}$ and $I_{\mathrm{TA}}$ given in Eqs. (24) and (25). Here $I_{\mathrm{LA}(\mathrm{TA})}$ quantifies the efficiency of the HEPs energy dissipation through the anharmonic interaction with LA (TA) phonon modes. In the limit of $T \rightarrow T_{\mathrm{c}}$ (that is, $\Delta \rightarrow 0$ ), $I_{\mathrm{LA}}$ vanishes (irrespective of $p$ ) but $I_{\mathrm{TA}}$ converges to a finite value as long as $p \neq 0$. Therefore, we obtain a nondiverging $\tau$ at $T_{\mathrm{c}}$ if $p \neq 0$, which readily follows from Eq. (22). When $p=0$, on the other hand, $I_{\mathrm{TA}} \equiv 0$ for arbitrary $T$ [because $I_{\mathrm{TA}} \propto w_{2}^{2}$; see Eq. (25)]. In this case, $I_{\mathrm{LA}}=I_{\mathrm{TA}}=0$ at $T_{\mathrm{c}}$ so that $\tau \rightarrow \infty$ at $T_{\mathrm{c}}$. We thus conclude that the anharmonic decay of HEPs to TA modes plays a prominent role in the efficient cooling of HEPs as well as in determining the significance of the $\tau$ divergence at $T_{\mathrm{c}}$.

Upon reducing the temperature to zero, the energy dissipation of HEPs gradually reduces due to monotonic decreases in $I_{\mathrm{LA}}$ and $I_{\mathrm{TA}}$ [see Fig. 3(c)]. The decrease in $I_{\mathrm{LA}(\mathrm{TA})}$ is attributed to the reduced phonon population, which results in a monotonic increase in $\tau$ at low $T$, as shown in Fig. 3(a). A similar increase has been found in various gapped systems, ${ }^{2-6,9-13,15,18}$ and it is attributable to the inefficient cooling of HEPs.

\section{B. Comparison with experimental data}

Now we apply the proposed theory to photoexcited carrier relaxation in quasi-one-dimensional (1D) $\mathrm{C}_{60}$ polymers. It was previously observed in experiments ${ }^{29}$ that the $\mathrm{C}_{60}$ polymers undergo the $\mathrm{CDW}$ transition $^{30}$ at $T_{\mathrm{c}}=60 \mathrm{~K}$, forming a well-defined energy gap at the Fermi level. This result implied the possibility of $\tau_{\text {exp }}$ divergence at $60 \mathrm{~K}$; nevertheless, optical pump-probe investigations ${ }^{18}$ of the $\mathrm{C}_{60}$ polymers revealed a monotonic variation in $\tau_{\exp }$ near $T_{\mathrm{c}}$, whose origin has yet to be clarified. This problem is solved by considering that the twisting phonon modes of the $\mathrm{C}_{60}$ polymer ${ }^{31}$ play the same role as the above-described TA modes. The only twisting mode is relevant despite the presence of many other TA modes in the system because these modes give quite minor contributions to the total phonon density of states. Figure 4(a) shows the numerical reproduction (indicated by lines) of the experimental data ${ }^{18}$ (indicated by circles) of the $T$-dependent $\tau_{\exp }$ of the $\mathrm{C}_{60}$ polymers. An overall agreement between the theory and the experiments is obtained by assuming $p \sim 0.3$. The other parameters we used are $\Omega_{\mathrm{TA}}=220 \mathrm{~cm}^{-1}$, $\Omega_{h}=360 \mathrm{~cm}^{-1}$, and $2 \Delta(0)=360 \mathrm{~K}$; the first two values were estimated from the phonon model for $1 \mathrm{D} \mathrm{C}_{60}$ polymers, ${ }^{31}$ and the last value gives $2 \Delta(0) / k_{B} T_{c}=6$ consistent with many CDW compounds. ${ }^{32}$

The generality of Eq. (22) is of great importance. The formula is applicable to $\tau_{\exp }$ in other gapped systems such as Tl-based cuprate superconductors ${ }^{2,6}$ and the solid fullerenes $\mathrm{K}_{3} \mathrm{C}_{60}$ and $\mathrm{Rb}_{3} \mathrm{C}_{60}$ (Ref. 3). Even these materials show a lack of $\tau_{\text {exp }}$ divergence; however, no theoretical studies have attempted to clarify their relaxation anomalies. We believe that the proposed theory will serve as a unified framework for nonequilibrium carrier dynamics in gapped systems.

To verify the flexibility of our model, we have compared our theory with the experimental data of Bi2212 which shows the divergence of $\tau_{\exp }$ at the $T_{\mathrm{c}}=75 \mathrm{~K}$ (Ref. 12), as shown in Fig. 4(b). A very good agreement between Eq. (22) and

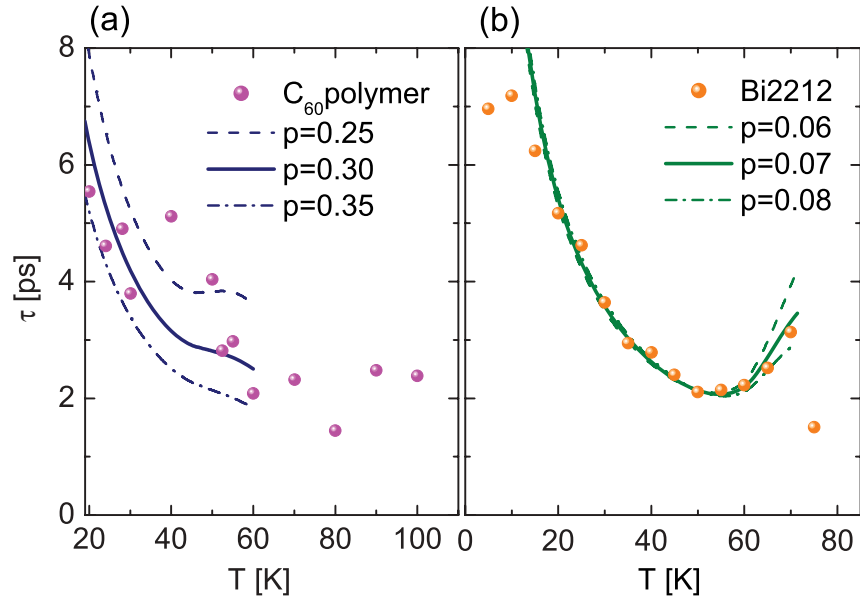

FIG. 4. (Color online) Experimental data of $\tau_{\exp }$ 's in (a) the $\mathrm{C}_{60}$ polymer (after Ref. 18) and (b) Bi2212 (after Ref. 12) and their numerical reproductions based on Eq. (22). The data for (a) $T \leqslant$ $T_{\mathrm{c}}=60 \mathrm{~K}$ and (b) $75 \mathrm{~K}$ collapse onto the theoretical curves with $p=0.3$ and $p=0.07$, respectively.

the data for Bi2212 can be obtained by setting $p=0.07$ which is smaller than $p=0.30$ in the $\mathrm{C}_{60}$ polymer case. The consistency for different two experimental data (for $\mathrm{C}_{60}$ polymers and $\mathrm{Bi} 2212$ ) supports the validity of our model. The larger $p$ for the $\mathrm{C}_{60}$ polymers is thought to originate from the curved geometry of the system. ${ }^{33,34}$ Finite surface curvature of the monoatomic carbon layer enhances the interatomic forces between neighboring carbon atoms, thus resulting in a significant increase in the magnitude of the aforementioned matrix element $w_{2}$ that is proportional to $p$ (Ref. 35).

\section{CONCLUSION}

In conclusion, we have developed a theory to describe the energy dissipation from HEPs $\left(\hbar \omega_{q, \mathrm{LA}}>2 \Delta\right)$ to LEPs $\left(\hbar \omega_{q, \mathrm{LA}}<2 \Delta\right)$ and TA modes in gapped systems below $T_{\mathrm{c}}$. This theory enables the evaluation of the $T$ dependence of $\tau$ as a function of the coupling strength $p \equiv w_{2} / w_{1}$ between the HEPs and the TA modes, and it explains the crossover between the diverging and the nondiverging behaviors of photoexcited carrier relaxation. The coupling between the HEPs and the TA mode also suggests the variation of $\tau_{\text {exp }}$ divergence in typical gapped systems. The quantitative estimation of the coupling strength for various materials ought to be a challenging task that we must tackle in the future.

\section{ACKNOWLEDGMENTS}

We thank K. Yakubo, Y. Asano, J. Onoe, K. Ohno, Y. Noda, and T. Matsuura for helpful discussions. This study was supported by a Grant-in-Aid for Scientific Research from the MEXT, Japan. H.S. acknowledges financial support from the Inamori Foundation and the Asahi Glass Foundation.

\section{APPENDIX : LIST OF COLLISION TERMS}

In this Appendix, we list $K_{A}^{(j)}(j=2,3), K_{B}^{(j)}(j=1,2,3)$, and $K_{\mathrm{LA}}$ defined by Eqs. (7), (8), and (11). 


\section{1. $K_{A}^{(2)}$}

There are two cases for the value of $K_{A}^{(2)}$ to be considered:

(i) $\Omega_{l}<\Omega_{\mathrm{TA}}$,

$$
\begin{gathered}
K_{A}^{(2)}=w_{2}^{2} \gamma_{2} G_{A}^{(2)} T\left(T^{\prime}-T\right) \\
G_{A}^{(2)}=\frac{1}{168}\left(\Omega_{\mathrm{TA}}^{7}-7 \Omega_{l}^{3} \Omega_{\mathrm{TA}}^{4}+7 \Omega_{l}^{4} \Omega_{\mathrm{TA}}^{3}-\Omega_{l}^{7}\right) ;
\end{gathered}
$$

(ii) $\Omega_{\mathrm{TA}}<\Omega_{l}$,

$$
\begin{gathered}
K_{A}^{(2)}=0 . \\
\text { 2. } K_{A}^{(3)}
\end{gathered}
$$

There also are two cases for the value of $K_{A}^{(3)}$ :

(i) $\Omega_{l}<\Omega_{\mathrm{TA}}$,

$$
K_{A}^{(3)}=K_{A}^{(2)}
$$

(ii) $\Omega_{\mathrm{TA}}<\Omega_{l}$

$$
K_{A}^{(3)}=0 .
$$$$
\text { 3. } K_{B}^{(1)}
$$

$K_{B}^{(1)}$ can be expressed by

$$
K_{B}^{(1)}=\sum_{j=1,2} Q_{B}^{(1)}(j)
$$

where $Q_{B}^{(1)}(1)$ and $Q_{B}^{(1)}(2)$ are given below: (i-1) $\Omega_{l}<\Omega_{\mathrm{TA}}<$ $\Omega_{h}$ and $\Omega_{l}+\Omega_{\mathrm{TA}}<\Omega_{h}$,

$$
\begin{gathered}
Q_{B}^{(1)}(1)=w_{2}^{2} \gamma_{1} G_{B}^{(1)}(1) T\left(T^{\prime}-T\right), \\
G_{B}^{(1)}(1)=\frac{71}{210} \Omega_{l}^{7}+\frac{1}{60}\left(6 \Omega_{l}^{2} \Omega_{\mathrm{TA}}^{5}+10 \Omega_{l}^{3} \Omega_{\mathrm{TA}}^{4}\right. \\
\left.+5 \Omega_{l}^{4} \Omega_{\mathrm{TA}}^{3}-21 \Omega_{l}^{7}\right),
\end{gathered}
$$

and

$$
\begin{gathered}
Q_{B}^{(1)}(2)=w_{2}^{2} \gamma_{1} G_{B}^{(1)}(2) T^{\prime}\left(T^{\prime}-T\right), \\
G_{B}^{(1)}(2)=\frac{\Omega_{\mathrm{TA}}^{4}\left(35 \Omega_{\mathrm{h}}^{3}-42 \Omega_{\mathrm{TA}} \Omega_{\mathrm{h}}^{2}+10 \Omega_{\mathrm{TA}}^{3}\right.}{420} \\
\left.-42 \Omega_{l}^{2} \Omega_{\mathrm{TA}}-35 \Omega_{l}^{3}\right) .
\end{gathered}
$$

(i-2) $\Omega_{l}<\Omega_{\mathrm{TA}}<\Omega_{h}$ and $\Omega_{h}<\Omega_{l}+\Omega_{\mathrm{TA}}$,

$$
Q_{B}^{(1)}(1)=w_{2}^{2} \gamma_{1} G_{B}^{(1)}(1) T\left(T^{\prime}-T\right),
$$

$$
\begin{aligned}
G_{B}^{(1)}(1)= & \frac{1}{420}\left(5 \Omega_{\mathrm{TA}}^{7}-35 \Omega_{h}^{3} \Omega_{\mathrm{TA}}^{4}+35 \Omega_{h}^{4} \Omega_{\mathrm{TA}}^{3}-5 \Omega_{h}^{7}\right. \\
& \left.+42 \Omega_{l}^{2} \Omega_{h}^{5}-70 \Omega_{l}^{3} \Omega_{h}^{4}+35 \Omega_{l}^{4} \Omega_{h}^{3}-7 \Omega_{l}^{7}\right),
\end{aligned}
$$

and

$$
\begin{gathered}
Q_{B}^{(1)}(2)=w_{2}^{2} \gamma_{1} G_{B}^{(1)}(2) T^{\prime}\left(T^{\prime}-T\right), \\
G_{B}^{(1)}(2)=\frac{1}{140}\left(\Omega_{h}-\Omega_{l}\right)^{5}\left(\Omega_{h}^{2}+5 \Omega_{l} \Omega_{h}+\Omega_{l}^{2}\right) .
\end{gathered}
$$

(ii-1) $\Omega_{\mathrm{TA}}<\Omega_{l}<\Omega_{h}$ and $\Omega_{l}+\Omega_{\mathrm{TA}}<\Omega_{h}$,

$$
Q_{B}^{(1)}(1)=w_{2}^{2} \gamma_{1} G_{B}^{(1)}(1) T\left(T^{\prime}-T\right),
$$

$$
G_{B}^{(1)}(1)=\frac{1}{420}\left(-5 \Omega_{\mathrm{TA}}^{7}+42 \Omega_{l}^{2} \Omega_{\mathrm{TA}}^{5}+105 \Omega_{l}^{3} \Omega_{\mathrm{TA}}^{4}\right),
$$

and

$$
Q_{B}^{(1)}(2)=w_{2}^{2} \gamma_{1} G_{B}^{(1)}(2) T^{\prime}\left(T^{\prime}-T\right),
$$

$$
\begin{aligned}
G_{B}^{(1)}(2)= & \frac{1}{420}\left[10 \Omega_{\mathrm{TA}}^{7}-42\left(\Omega_{h}^{2}+\Omega_{l}^{2}\right) \Omega_{\mathrm{TA}}^{5}\right. \\
& \left.+35\left(\Omega_{h}^{3}-\Omega_{l}^{3}\right) \Omega_{\mathrm{TA}}^{4}\right] .
\end{aligned}
$$

(ii-2) $\Omega_{\mathrm{TA}}<\Omega_{l}<\Omega_{h}$ and $\Omega_{h}<\Omega_{l}+\Omega_{\mathrm{TA}}$,

$$
Q_{B}^{(1)}(1)=w_{2}^{2} \gamma_{1} G_{B}^{(1)}(1) T\left(T^{\prime}-T\right),
$$

$$
\begin{aligned}
G_{B}^{(1)}(1)= & \frac{1}{420}\left[-35\left(\Omega_{h}^{3}-\Omega_{l}^{3}\right) \Omega_{\mathrm{TA}}^{4}+35\left(\Omega_{h}^{4}-\Omega_{l}^{4}\right) \Omega_{\mathrm{TA}}^{3}\right. \\
& \left.-5 \Omega_{h}^{7}+42 \Omega_{l}^{2} \Omega_{h}^{5}-70 \Omega_{l}^{3} \Omega_{h}^{4}+35 \Omega_{l}^{4} \Omega_{h}^{3}-2 \Omega_{l}^{7}\right],
\end{aligned}
$$

and

$$
\begin{gathered}
Q_{B}^{(1)}(2)=w_{2}^{2} \gamma_{1} G_{B}^{(1)}(2) T^{\prime}\left(T^{\prime}-T\right), \\
G_{B}^{(1)}(2)=\frac{1}{140}\left(\Omega_{h}-\Omega_{l}\right)^{5}\left(\Omega_{h}^{2}+5 \Omega_{l} \Omega_{h}+\Omega_{l}^{2}\right) . \\
\text { 4. } K_{B}^{(2)}
\end{gathered}
$$

Next, we show the expressions of $K_{B}^{(2)}$. There are five cases to distinguish:

(i-1) $\Omega_{l}<\Omega_{\mathrm{TA}}<\Omega_{h}$ and $\Omega_{h}<2 \Omega_{\mathrm{TA}}$,

$$
K_{B}^{(2)}=w_{2}^{2} \gamma_{2} G_{B}^{(2)} T\left(T^{\prime}-T\right),
$$

$G_{B}^{(2)}=\frac{1}{420}\left(3 \Omega_{\mathrm{TA}}^{7}-35 \Omega_{h}^{4} \Omega_{\mathrm{TA}}^{3}+42 \Omega_{h}^{5} \Omega_{\mathrm{TA}}^{2}-5 \Omega_{h}^{7}-5 \Omega_{l}^{7}\right)$.

(i-2) $\Omega_{l}<\Omega_{\mathrm{TA}}<\Omega_{h}$ and $2 \Omega_{\mathrm{TA}}<\Omega_{h}$,

$$
\begin{aligned}
K_{B}^{(2)} & =w_{2}^{2} \gamma_{2} G_{B}^{(2)} T\left(T^{\prime}-T\right), \\
G_{B}^{(2)} & =\frac{1}{420}\left(147 \Omega_{\mathrm{TA}}^{7}-5 \Omega_{l}^{7}\right) .
\end{aligned}
$$

(ii-1) $\Omega_{\mathrm{TA}}<\Omega_{l}<\Omega_{h}$ and $\Omega_{h}<2 \Omega_{\mathrm{TA}}$,

$$
K_{B}^{(2)}=w_{2}^{2} \gamma_{2} G_{B}^{(2)} T\left(T^{\prime}-T\right),
$$

$$
\begin{aligned}
G_{B}^{(2)}= & \frac{1}{420}\left[-35\left(\Omega_{h}^{4}-\Omega_{l}^{4}\right) \Omega_{\mathrm{TA}}^{3}-42\left(\Omega_{h}^{5}-\Omega_{l}^{5}\right) \Omega_{\mathrm{TA}}^{2}\right. \\
& \left.+5\left(\Omega_{h}^{7}-\Omega_{l}^{7}\right)\right] .
\end{aligned}
$$

(ii-2) $\Omega_{\mathrm{TA}}<\Omega_{l}<\Omega_{h}$ and $2 \Omega_{\mathrm{TA}}<\Omega_{h}$,

$$
K_{B}^{(2)}=w_{2}^{2} \gamma_{2} G_{B}^{(2)} T\left(T^{\prime}-T\right),
$$

$$
G_{B}^{(2)}=\frac{1}{420}\left(144 \Omega_{\mathrm{TA}}^{7}+35 \Omega_{l}^{4} \Omega_{\mathrm{TA}}^{3}-42 \Omega_{l}^{5} \Omega_{\mathrm{TA}}^{2}+5 \Omega_{l}^{7}\right) \text {. }
$$


(iii) $2 \Omega_{\mathrm{TA}}<\Omega_{l}$,

$$
K_{B}^{(2)}=0
$$

$$
\text { 5. } K_{B}^{(3)}
$$

For $K_{B}^{(3)}$, there are two cases:

(i) $\Omega_{l}<\Omega_{\mathrm{TA}}$,

$$
\begin{aligned}
K_{B}^{(3)}=w_{2}^{2} \gamma_{2} G_{B}^{(3)} T\left(T^{\prime}-T\right), \\
G_{B}^{(3)}=\frac{(-1)}{420}\left(2 \Omega_{\mathrm{TA}}^{7}-35 \Omega_{l}^{3} \Omega_{\mathrm{TA}}^{4}+70 \Omega_{l}^{4} \Omega_{\mathrm{TA}}^{3}\right. \\
\left.-42 \Omega_{l}^{5} \Omega_{\mathrm{TA}}^{2}+5 \Omega_{l}^{7}\right) .
\end{aligned}
$$

(ii) $\Omega_{\mathrm{TA}}<\Omega_{l}$

$$
K_{B}^{(3)}=0 .
$$

Finally, using Eqs. (11)-(21), (23), and (A1)-(A34), we obtain $I_{\mathrm{TA}}$ defined by Eq. (25).

\section{6. $K_{\mathrm{LA}}$}

In this section, we present the expression of $I_{\mathrm{LA}}$ in Eq. (24). As with Eq. (8), the integrand in Eq. (7) depends on the phonon frequencies $\omega$ and $\omega^{\prime}$. We find that there are two cases to distinguish: (i) $2 \Omega_{l}<\Omega_{h}$ and (ii) $\Omega_{h}<2 \Omega_{l}$. The integral in Eq. (7) is written as

$$
K_{\mathrm{LA}}=w_{1}^{2} \gamma_{0} G_{C}^{(1)} T\left(T^{\prime}-T\right)+w_{1}^{2} \gamma_{0} G_{C}^{(2)} T^{\prime}\left(T^{\prime}-T\right) .
$$

For case (i), $G_{C}^{(1)}$ and $G_{C}^{(2)}$ are given by

$$
\begin{gathered}
G_{C}^{(1)}=\frac{71}{210} \Omega_{l}^{7}, \\
G_{C}^{(2)}=\frac{\Omega_{l}^{4}}{420}\left(35 \Omega_{h}^{3}-42 \Omega_{l} \Omega_{h}^{2}-67 \Omega_{l}^{3}\right) .
\end{gathered}
$$

For case (ii)

$$
\begin{gathered}
G_{C}^{(1)}=\frac{1}{420}\left(-5 \Omega_{h}^{7}+42 \Omega_{l}^{2} \Omega_{h}^{5}-35 \Omega_{l}^{3} \Omega_{h}^{4}-2 \Omega_{l}^{7}\right), \\
G_{C}^{(2)}=\frac{1}{140}\left(\Omega_{h}-\Omega_{l}\right)^{5}\left(\Omega_{h}^{2}+5 \Omega_{l} \Omega_{h}+\Omega_{l}^{2}\right) .
\end{gathered}
$$

*shota-o@ynu.ac.jp

${ }^{1}$ V. V. Kabanov, J. Demsar, B. Podobnik, and D. Mihailovic, Phys. Rev. B 59, 1497 (1999).

${ }^{2}$ D. C. Smith, P. Gay, D. Z. Wang, J. H. Wang, Z. F. Ren, and J. F. Ryan, Physica C (Amsterdam) 341, 2219 (2000).

${ }^{3}$ S. B. Fleischer, B. Pevzner, D. J. Dougherty, H. J. Zeiger, G. Dresselhaus, M. S. Dresselhaus, E. P. Ippen, and A. F. Hebard, Phys. Rev. B 62, 1366 (2000).

${ }^{4}$ V. V. Kabanov, J. Demsar, and D. Mihailovic, Phys. Rev. Lett. 95, 147002 (2005).

${ }^{5}$ R. P. S. M. Lobo, J. D. LaVeigne, D. H. Reitze, D. B. Tanner, Z. H. Barber, E. Jacques, P. Bosland, M. J. Burns, and G. L. Carr, Phys. Rev. B 72, 024510 (2005).

${ }^{6}$ E. E. M. Chia, J.-X. Zhu, D. Talbayev, R. D. Averitt, A. J. Taylor, K.-H. Oh, I.-S. Jo, and S.-I. Lee, Phys. Rev. Lett. 99, 147008 (2007). ${ }^{7}$ T. Mertelj, P. Kusar, V. V. Kabanov, L. Stojchevska, N. D. Zhigadlo, S. Katrych, Z. Bukowski, J. Karpinski, S. Weyeneth, and D. Mihailovic, Phys. Rev. B 81, 224504 (2010).

${ }^{8}$ C. W. Luo, H. P. Lo, C. H. Su, I. H. Wu, Y.-J. Chen, K. H. Wu, J.-Y. Lin, T. M. Uen, J. Y. Juang, and T. Kobayashi, Phys. Rev. B 82, 104512 (2010).

${ }^{9}$ S. K. Nair, X. Zou, E. E. M. Chia, J.-X. Zhu, C. Panagopoulos, S. Ishida, and S. Uchida, Phys. Rev. B 82, 212503 (2010).

${ }^{10}$ E. E. M. Chia, D. Talbayev, J.-X. Zhu, J. D. Thompson, A. J. Taylor, H. Q. Yuan, T. Park, C. Panagopoulos, G. F. Chen, J. L. Luo, and N. L. Wang, Phys. Rev. Lett. 104, 027003 (2010).

${ }^{11}$ G. Coslovich, C. Giannetti, F. Cilento, S. Dal Conte, G. Ferrini, P. Galinetto, M. Greven, H. Eisaki, M. Raichle, R. Liang, A. Damascelli, and F. Parmigiani, Phys. Rev. B 83, 064519 (2011).

${ }^{12}$ Y. Toda, T. Mertelj, P. Kusar, T. Kurosawa, M. Oda, M. Ido, and D. Mihailovic, Phys. Rev. B 84, 174516 (2011).

${ }^{13}$ Y. H. Ren, Y. Gong, T. Nosach, J. Li, J. J. Tu, L. J. Li, G. H. Cao, and Z. A. Xu, J. Appl. Phys. 111, 07 E134 (2012).

${ }^{14}$ J. Demsar, K. Biljaković, and D. Mihailovic, Phys. Rev. Lett. 83, 800 (1999).
${ }^{15}$ E. E. M. Chia, J.-X. Zhu, H. J. Lee, N. Hur, N. O. Moreno, E. D. Bauer, T. Durakiewicz, R. D. Averitt, J. L. Sarrao, and A. J. Taylor, Phys. Rev. B 74, 140409(R) (2006).

${ }^{16}$ K. Shimatake, Y. Toda, and S. Tanda, Phys. Rev. B 73, 153403 (2006); 75, 115120 (2007).

${ }^{17}$ R. V. Yusupov, T. Mertelj, J.-H. Chu, I. R. Fisher, and D. Mihailovic, Phys. Rev. Lett. 101, 246402 (2008).

${ }^{18}$ Y. Toda, S. Ryuzaki, and J. Onoe, Appl. Phys. Lett. 92, 094102 (2008).

${ }^{19}$ S. Watanabe, R. Kondo, S. Kagoshima, and R. Shimano, Phys. Rev. B 80, 220408(R) (2009).

${ }^{20} \mathrm{We}$ focus on $\tau$ only below $T_{\mathrm{c}}$. Above $T_{\mathrm{c}}$, on the other hand, the phonon bottleneck concept is violated because of the absence of the energy gap: The relaxation dynamics is governed by the electron-electron and electron-phonon collisions (Refs. 21 and 22).

${ }^{21}$ P. B. Allen, Phys. Rev. Lett. 59, 1460 (1987).

${ }^{22}$ V. V. Kabanov and A. S. Alexandrov, Phys. Rev. B 78, 174514 (2008).

${ }^{23}$ A. Rothwarf and B. N. Taylor, Phys. Rev. Lett. 19, 27 (1967).

${ }^{24} \mathrm{~A}$ similar LA-TA coupling in Ge crystal has been discussed by the authors of Ref. 25.

${ }^{25}$ K. Okubo and S. I. Tamura, Phys. Rev. B 28, 4847 (1983).

${ }^{26}$ J. M. Ziman, Electrons and Phonons (Oxford University Press, New York, 2001).

${ }^{27}$ For example, the first term in the curly brackets in $S_{B}$ implies that one phonon with $\omega_{2}$ decays into two phonons with $\omega_{0}$ and $\omega_{1}$.

${ }^{28}$ In the calculation, we assume that $T^{\prime}$ is fixed [that is, $T^{\prime}(0) \simeq$ $T^{\prime}(\infty)$ ] to derive the analytical expression of $\tau$. A similar assumption has been used by the authors of Ref. 1 for the case of the absence of the TA mode.

${ }^{29}$ J. Onoe, A. Takashima, and Y. Toda, Appl. Phys. Lett. 97, 241911 (2010).

${ }^{30}$ S. Ono and H. Shima, Europhys. Lett. 96, 27011 (2011). 
${ }^{31}$ S. Ono and H. Shima, J. Phys. Soc. Jpn. 80, 064704 (2011).

${ }^{32}$ G. Grüner, Density Waves in Solids (Addison-Wesley, Reading, MA, 1994).

${ }^{33}$ Y. Noda and K. Ohno, Synthetic Met. 161, 1546 (2011).
${ }^{34}$ J. Onoe, T. Ito, H. Shima, H. Yoshioka, and S. Kimura, Europhys. Lett. 98, 27001 (2012).

${ }^{35}$ The matrix elements would be derived from a first-principles calculation with regard to the anharmonic force constants (Ref. 36).

${ }^{36}$ K. Esfarjani and H. T. Stokes, Phys. Rev. B 77, 144112 (2008). 